\title{
REAL ANALYTICITY OF HOMEOMORPHIC CR MAPPINGS BETWEEN REAL ANALYTIC HYPERSURFACES IN $\mathbf{C}^{2}$
}

\author{
YIFEI PAN
}

(Communicated by Eric Bedford)

ABSTRACT. In this note, we prove a real analyticity result for smooth CR homeomorphisms in $\mathbf{C}^{2}$.

\section{INTRODUCTION}

In this paper we prove the following main result.

Theorem 1. Let $M_{1}$ and $M_{2}$ be smooth real analytic hypersurfaces in $\mathbf{C}^{2}$ with $M_{1}$ not Levi falt. If $f: M_{1} \rightarrow M_{2}$ is a $C^{\infty}$ smooth homeomorphic CR mapping that extends holomorphically to one side of $M_{1}$, then $f$ extends holomorphically to a neighborhood of $M_{1}$ in $\mathbf{C}^{2}$. Furthermore, if $M_{1}$ is of infinite type (resp. finite type) at $p \in M_{1}$, then $M_{2}$ is also of infinite type (resp. finite type) at $f(p)$.

For $M_{1}$ and $M_{2}$ pseudoconvex and of finite type, many more general theorems of this type were proved in Bell [B] and Bell and Catlin [BC]. Our result is a consequence of a general reflection principle of Baouendi and Rothschild [BR2] and a unique continuation theorem for holomorphic mappings that we shall prove. We first state the general reflection principle of Baouendi and Rothschild [BR2].

Theorem 2 (Baouendi-Rothschild). Let $M_{1}$ and $M_{2}$ be smooth real analytic hypersurfaces in $\mathbf{C}^{2}$. Let $f: M_{1} \rightarrow M_{2}$ be a $C^{\infty} \quad C R$ mapping that extends holomorphically to one side of $M_{1}$. If $M_{1}$ is not Levi flat and the Jacobian determinant of $f$ does not vanish to infinite order at a point $p \in M_{1}$, then $f$ extends holomorphically to a neighborhood of $p \in M_{1}$ in $\mathbf{C}^{2}$.

As one can see in the proof of Theorem 2 in [BR2], the same result remains true as long as the transversal component of the map does not vanish to infinite order at a point of extendability. From this remark, to prove Theorem 1, it suffices to prove that if $M_{1}$ is not Levi flat and $f: M_{1} \rightarrow M_{2}$ is a CR homeomorphism, then the Jacobian determinant of $f$ does not vanish to infinite order at every point of $M_{1}$. This amounts to proving a unique continuation theorem when $M_{1}$ is of infinite type and to using a generalized Hopf Lemma by Baouendi and Rothschild [BR1] when $M_{1}$ is of finite type. The following unique continuation result will be proved in this paper.

Received by the editors January 9, 1993 and, in revised form, April 22, 1993.

1991 Mathematics Subject Classification. Primary 32H35. 
Theorem 3. Let $M_{1}$ and $M_{2}$ be smooth hypersurfaces in $\mathbf{C}^{m}$ and $\mathbf{C}^{n}$, respectively, and let $f: M_{1} \rightarrow M_{2}$ be a continuous $C R$ mapping that extends holomorphically to one side of $M_{1}$, say $M_{1}^{-}$. If $f$ vanishes to infinite order at $p \in M_{1}$ and $M_{2}$ contains a complex hypersurface through $f(p)$, then $f\left(M_{1} \cup M_{1}^{-}\right) \subset M_{2}$.

This result extends a theorem of Bell and Lempert [BL] in which $M_{2}$ was assumed to be a Levi flat hypersurface.

The proof of Theorem 3 is contained in the next section. We prove some invariants under $\mathrm{CR}$ homeomorphisms which are needed in the proof of Theorem 1 . The proof of Theorem 1 will be given in $\S 3$.

\section{UNIQUE CONTINUATION FOR CR MAPPINGS}

To prove our results, we have to prove a unique continuation theorem when $M_{1}$ is of infinite type which is slightly more general than Theorem 3.

Theorem 4. Let $M_{1}$ and $M_{2}$ be smooth hypersurfaces in $\mathbf{C}^{m}$ and $\mathbf{C}^{n}$, respectively, and let $f: M_{1} \rightarrow M_{2}$ be a continuous $C R$ mapping that extends holomorphically to one side of $M_{1}$, say $M_{1}^{-}$. If the transversal component of $f$ vanishes to infinite order at $p \in M_{1}$ and $M_{2}$ contains a complex hypersurface through $f(p)$, then $f\left(M_{1} \cup M_{1}^{-}\right) \subset M_{2}$.

Remarks. (1) A theorem of this kind was first proved by Bell and Lempert in [BL] where $M_{2}$ was assumed to be Levi flat. Their methods led to proving other results on unique continuation for holomorphic mappings.

(2) In [BR1], Baouendi and Rothschild proved a generalized complex Hopf Lemma which has proved very useful and will be used in our proof of Theorem 1 when $M_{1}$ is of finite type. To state it we introduce some notation. If $H$ is a smooth CR mapping between hypersurfaces $M_{1}$ and $M_{2}$ in $\mathrm{C}^{n}$, we denote by Jac $H$ the Jocobian determinant of $H$ considered as a mapping from the real manifold $M_{1}$ to the real manifold $M_{2}$. A hypersurface is said to be minimal at a point if it does not contain a complex hypersurface through the point. For the notion of minimal convexity at a point, see [BR1] for definitions.

(3) We give an example from [BR1] to show that a CR homeomorphism does not have to be a diffeomorphism. Let $M$ be the hypersurface in $\mathrm{C}^{2}$ given by $\bar{w}=w e^{i|z|^{2}}$. It is easy to check that this equation does define a real analytic hypersurface which is neither of finite type nor Levi flat at 0 . The mapping $(z, w) \rightarrow\left(\sqrt{3} z, w^{3}\right)$ restricts to a CR self map of $M$ which is a CR homeomorphism. But the transversal component of the map vanishes to third order at 0 , and hence the map is not a diffeomorphism. An example of this kind was observed by Bell [B] when $M$ is Levi flat: $\left(z, w^{3}\right): M \rightarrow M$ where $M=\{\operatorname{Im} w=0\}$.

Theorem 5 (Baouendi-Rothschild). Suppose that $M_{1}$ is minimal at $p$ and $\operatorname{Jac} H$ $\not \equiv 0$. If $M_{2}$ is minimally convex at $H(p)$, then the differential of $H$ at $p$ is nonzero.

What we really proved in Theorem 1 is that if $f$ vanishes to infinite order, then the transversal component of $f$ is identically zero. On the other hand, it is easy to see that if $\mathrm{Jac} H \not \equiv 0$, then $f\left(M_{1} \cup M_{1}^{-}\right) \not \subset M_{2}$. Hence, we could restate our theorem as follows. 
Theorem 6. Let $H: M_{1} \rightarrow M_{2}$ be a smooth $C R$ mapping that extends holomorphically to one side of $M_{1}$. If $M_{2}$ is not minimal at $H(p)$ and $\mathrm{Jac} H \not \equiv 0$, then the differential of $H$ vanishes to at most finite order at $p$.

Now we can see that our result can be regarded as a substitute for the generalized complex Hopf lemma of Baouendi and Rothschild when the hypersurface $M_{2}$ is not minimal, i.e., when $M_{2}$ contains a complex hypersurface.

To prove Theorem 4, we need a unique continuation theorem of one variable.

Lemma 7. Let $U$ be the upper half disc in the plane, and let $f(z)=u(z)+i v(z)$ be a function holomorphic in $U$ and continuous up to the real axis $(f(0)=0)$. If $|v(x)| \leq|u(x)|$ on the real axis and $f$ vanishes to infinite order at the origin, i.e., $|f(z)| \leq C_{N}|z|^{N}$ for each positive integer $N$, then $f$ is identically zero.

Proof. This lemma is a consequence of a unique continuation lemma proved in [HKMP], which says if $u(x) \geq 0$ on the real axis, then $f$ is identically zero. To prove Lemma 7, we consider the holomorphic function $f^{2}(z)=u^{2}(z)-$ $v^{2}(z)+2 i u(z) v(z)$. If $|v(x)| \leq|u(x)|$ on the real axis, then the real part of $f^{2}(z)$ is nonnegative on the real axis and $f^{2}(z)$ also vanishes to infinite order at the origin as $f$ does. Hence, we conclude that $f^{2}(z)$ is zero and so is $f$. The proof of the lemma is complete.

Proof of Theorem 4. By considering one-dimensional complex slices which cut the hypersurface $M_{1}$ transversally, it suffices to prove Theorem 4 in the simplified case that $m=1, M_{1}$ is equal to the real axis, $p=0$, and $M_{1}^{-}$is the upper half disc $U$ in the plane. Hence, from this point forward, we will be studying a holomorphic mapping $f$ on the upper half disc $U$ into $\mathbf{C}^{n}$ which extends continuously to the real axis and which maps the real axis into $M_{2}$. Now by a linear change of coordinates at $f(0)$ for $M_{2}$, we may assume that $f(0)=0$ and $M_{2}$ is given near 0 by $\operatorname{Im} z_{n}=h\left(\operatorname{Re} z_{n}, z_{1}, \ldots, z_{n-1}\right)$ where $h(0)=\nabla h(0)=0$. Since $M_{2}$ contains a complex hypersurface through 0 , it follows that $h\left(z_{1}, \ldots, z_{n-1}, 0\right) \equiv 0$ for all $\left(z_{1}, \ldots, z_{n-1}, 0\right)$. After a biholomorphic change of coordinates, we can assume that $M_{2}$ is given by the equation $\operatorname{Im} z_{n}=\operatorname{Re} z_{n} g\left(\operatorname{Re} z_{n}, z_{1}, \ldots, z_{n-1}\right)$ where $g(0)=0$.

Now let $f(\zeta)=\left(f_{1}(\zeta), \ldots, f_{n-1}(\zeta), f_{n}(\zeta)\right)$ be a holomorphic mapping from $U$ to $M_{2}$ that maps the real axis to $M_{2}$. Then

$$
\operatorname{Im} f_{n}(\zeta)=\operatorname{Re} f_{n}(\zeta) g\left(\operatorname{Re} f_{n}(\zeta), f_{1}(\zeta), \ldots, f_{n-1}(\zeta)\right)
$$

when $z$ is real. From this and then for small $x$ near 0 , we have

$$
\left|\operatorname{Im} f_{n}(x)\right| \leq\left|\operatorname{Re} f_{n}(x)\right| .
$$

This says that the function $f_{n}(\zeta)$ satisfies the conditions of Lemma 7. Therefore, $f_{n}(\zeta) \equiv 0$. This implies that $f\left(M_{1} \cup M_{1}^{-}\right) \subset M_{2}$. The proof of Theorem 4 is complete.

\section{CR INVARIANTS OF A HYPERSURFACE}

In this section we prove some results on $\mathrm{CR}$ invariants of a hypersurface that are needed in the proof of Theorem 1. However, these results are of interest in their own right. Actually we will prove that for a hypersurface, a point being Levi flat is CR invariant under CR homeomorphisms and so is the number of 
nonzero eigenvalues of the Levi form at a point under CR diffeomorphisms. Let $M$ be a smooth hypersurface in $\mathbf{C}^{n}$. Let $r$ be the defining function for $M$, i.e., $M=\{r=0\}$ where $d r \neq 0$ on $M$; and we denote throughout $M^{-}=\left\{z \in C^{n} ; r<0\right\}$. The Levi form of the real hypersurface $M$ at a point $p \in M$ is defined to be the complex hessian of its defining function acting on the maximal complex tangential space $T_{p}^{C} M$ to $M$. To be precise, the Levi form, $L_{r}$, is defined as follows:

$$
L_{r}(v, \bar{v})=\sum_{i j=1}^{n} \frac{\partial^{2} r}{\partial z_{i} \partial \bar{z}_{j}} v_{i} \bar{v}_{j},
$$

where $v \in T_{p}^{C} M$.

A point $p$ in $M$ is said to be Levi flat if the Levi form of $M$ at $p$ vanishes identically. A real hypersurface $M$ is said to be Levi flat if its Levi form vanishes identically at every point.

We prove that a point being Levi flat is $\mathrm{CR}$ invariant and so is the number of nonzero eigenvalues of the Levi form.

Theorem 8. Let $M_{1}$ and $M_{2}$ be $C^{2}$ smooth hypersurfaces in $\mathbf{C}^{n}$, and let $f$ : $M_{1} \rightarrow M_{2}$ be a $C^{1}$ smooth CR homeomorphism. If $p \in M_{1}$ is a Levi flat point of $M_{1}$, then $f(p)$ is a Levi flat point of $M_{2}$. Furthermore, the number of nonzero eigenvalues of the Levi form of $M_{1}$ at a point $q$ is the same as that of $M_{2}$ at $f(q)$ if $f$ is further assumed to be a diffeomorphism.

We also consider holomorphic mappings which map a real hypersurface into a Levi flat hypersurface. Bell and Lempert [BL] have shown that such mappings are regular up to $M$ in a flattened complex normal direction for a Levi flat hypersurface.

Theorem 9. Let $M_{1}$ and $M_{2}$ be $C^{2}$ smooth real hypersurfaces in $\mathbf{C}^{n}$ and $\mathbf{C}^{m}$, respectively, with $M_{1}$ not Levi flat and $M_{2}$ Levi flat. Suppose that $f: M_{1} \rightarrow M_{2}$ is a $C^{1}$ smooth $C R$ mapping that extends holomorphically to one side of $M_{1}$, say $M_{1}^{-}$; then we have $F\left(M_{1} \cup M_{1}^{-}\right) \subset M_{2}$.

An easy consequence of this result is that Levi flatness is CR invariant. Using this result we can also prove that a hypersurface that is not Levi flat is also invariant under $\mathrm{CR}$ homeomorphisms.

Theorem 10. Let $f: M_{1} \rightarrow M_{2}$ be a $C^{1}$ smooth $C R$ homeomorphism. If $M_{1}$ is not Levi flat, neither is $M_{2}$.

Theorems 8 and 9 will be used in proving Theorem 1 . We point out that the proofs of Theorems 8-10 are largely based on an identity which shows that the Levi forms of real hypersurfaces under a CR mapping and the degeneracy of the map can be tied together.

Lemma 12. Let $M_{1}$ and $M_{2}$ be $C^{2}$ smooth real hypersurfaces in $\mathbf{C}^{n}$ and $\mathbf{C}^{m}$, respectively. Suppose that $f: M_{1} \rightarrow M_{2}$ is a $C^{1}$ smooth $C R$ mapping and that $r$ and $\rho$ are the defining functions of $M_{1}$ and $M_{2}$, respectively. Let $z_{0} \in M$ with $r_{n}\left(z_{0}\right)=\frac{\partial}{\partial z_{n}} r\left(z_{0}\right) \neq 0$. Then for $z$ near $z_{0}$ on $M_{1}$, we have the following identity:

$$
L_{\rho} \circ f\left(f^{\prime}(z) v, \overline{\left.f^{\prime}(z) v\right)}=\frac{1}{r_{n}(z)}\left\{\sum_{i=1}^{m} \frac{\partial \rho}{\partial w_{i}} \circ f \frac{\partial f_{i}}{\partial z_{n}}\right\} L_{r}(v, \bar{v})\right.
$$


where $v \in T_{z}^{C} M_{1}, f=\left(f_{1}, \ldots, f_{m}\right)$, and $L_{\rho} \circ f$ is the Levi form of $M_{2}$ at $f(z)$.

Proof. Since $r_{n}(z) \neq 0$ for $z$ near $z_{0}$ on $M_{1}$, the complex tangential space has a basis consisting of

$$
L_{k}=r_{n} \frac{\partial}{\partial z_{k}}-r_{k} \frac{\partial}{\partial z_{n}}
$$

for $k=1, \ldots, n-1$. To show (1), it suffices to show that it is true for the basis. Since $f$ is $\mathrm{CR}$, then we have

$$
\bar{L}_{k} f_{j}(z)=0 \quad \text { for } k=1, \ldots, n-1, j=1, \ldots, m .
$$

It follows from $f: M_{1} \rightarrow M_{2}$ that we have on $M_{1}$

$$
\rho \circ f(z)=0 \text {. }
$$

Taking the complex tangential derivative $L_{k}$ on (2), it follows that

$$
\sum_{i=1}^{m} \frac{\partial \rho}{\partial w_{i}} \circ f L_{k} f_{i}=0
$$

Taking $\overline{L_{k}}$ on (3), we get

$$
\sum_{i j}^{m} \frac{\partial^{2} \rho}{\partial w_{i} \overline{\partial w_{j}}} \circ f\left(L_{k} f_{i}\right)\left(\overline{L_{k} f_{j}}\right)+\sum_{i=1}^{m} \frac{\partial}{\partial w_{i}} \circ f \bar{L}_{k} L_{k} f_{i}=0 .
$$

In order to show (1), it remains to rewrite (4). We identify the vector fields $L_{k}$ with the vectors $v_{k}=\left[0, \ldots, r_{n}, \ldots,-r_{k}\right]$. First we notice that the Levi form acting on $L_{k}$ has the form

$$
L_{r}\left(v_{k}, \bar{v}_{k}\right)=r_{\bar{n}}\left(r_{n} r_{k \bar{k}}-r_{k} r_{n \bar{k}}\right)-r_{\bar{k}}\left(r_{n} r_{k \bar{n}}-r_{k} r_{n \bar{n}}\right) .
$$

Secondly we have

$$
\begin{aligned}
\overline{L_{k}} L_{k} f_{i} & =\left(r_{\bar{n}} \frac{\partial}{\partial \bar{z}_{k}}-r_{\bar{k}} \frac{\partial}{\partial \bar{z}_{n}}\right)\left(r_{n} \frac{\partial f_{i}}{\partial z_{k}}-r_{k} \frac{\partial f_{i}}{\partial z_{n}}\right) \\
& =r_{\bar{n}} r_{n \bar{k}} \frac{\partial f_{i}}{\partial z_{k}}-r_{\bar{n}} r_{k \bar{k}} \frac{\partial f_{i}}{\partial z_{n}}-r_{\bar{k}} r_{n \bar{n}} \frac{\partial f_{i}}{\partial z_{k}}+r_{\bar{k}} r_{k \bar{n}} \frac{\partial f_{i}}{\partial z_{n}} .
\end{aligned}
$$

It follows from (6) that

$$
\begin{aligned}
\sum_{i=1}^{m} \frac{\partial \rho}{\partial w_{i}} \circ f_{i} \overline{L_{k}} L_{k} f_{i} \\
=r_{\bar{n}} r_{n \bar{k}} \sum_{i=1}^{m} \frac{\partial \rho}{\partial w_{i}} \circ f \frac{\partial f_{i}}{\partial z_{k}}-r_{\bar{n}} r_{k \bar{k}} \sum_{i=1}^{m} \frac{\partial \rho}{\partial w_{i}} \circ f \frac{\partial f_{i}}{\partial z_{n}} \\
\quad-r_{\bar{k}} r_{n \bar{n}} \sum_{i=1}^{m} \frac{\partial \rho}{\partial w_{i}} \circ f \frac{\partial f_{k}}{\partial z_{k}}+r_{\bar{k}} r_{k \bar{n}} \sum_{i=1}^{m} \frac{\partial \rho}{\partial w_{i}} \circ f \frac{\partial f_{k}}{\partial z_{n}} \\
=\frac{1}{r_{n}}\left\{\sum_{j=1}^{m} \frac{\partial \rho}{\partial w_{j}} \circ f \frac{\partial f_{j}}{\partial z_{n}}\right\} L_{r}\left(L_{k}, \bar{L}_{k}\right),
\end{aligned}
$$


where we have used (5) and the identity

$$
r_{n}\left\{\sum_{i=1}^{m} \frac{\partial \rho}{\partial w_{i}} \circ f \frac{\partial f_{i}}{\partial z_{k}}\right\}=r_{k}\left\{\sum_{i=1}^{m} \frac{\partial \rho}{\partial w_{i}} \circ f \frac{\partial f_{i}}{\partial z_{n}}\right\},
$$

which is a rewritten form of (3). Finally, we note that

$$
f^{\prime}(z) v_{k}=\left(L_{k} f_{1}, \ldots, L_{k} f_{m}\right)
$$

where $v_{k}$ is the vector form of $L_{k}$ as defined earlier. Combining (4), (7), and (8) gives the proof of Lemma 4.

Proof of Theorem 8. If $p$ is a Levi flat point of $M_{1}$, then by Lemma 12 we have $L_{\rho} \circ f\left(f^{\prime} v, \overline{f^{\prime} v}\right)=0$ for all $v \in T_{p}^{C} M_{1}$. Since $f: M_{1} \rightarrow M_{2}$ is a CR homeomorphism, $f^{\prime}(p): T_{p}^{C} M_{1} \rightarrow T_{f(p)}^{C} M_{2}$ is a nonsingular linear transformation, and hence, the Levi form $L \rho$ of $M_{2}$ at $f(p)$ is identically zero. This implies that $f(p)$ is a Levi flat point. To prove the second statement of Theorem 8 , we notice that if $f$ is a diffeomorphism, then the constant appearing in front of the Levi form in Lemma 12 is not zero and so the number of nonzero eigenvalues of $M_{1}$ at $p$ is equal to that of $M_{2}$ at $f(p)$. Actually what we have proven is that the set of eigenvalues of $M_{1}$ at $p$ is proportional to that of $M_{2}$ at $f(p)$.

Proof of Theorem 9. We pick a point on $M_{1}$ such that the Levi form is not zero identically at $p$ and, hence, in a neighborhood, say $U$, of $p$ in $M_{1}$. Let $p_{0}$ be a point in $U$. By a linear change of coordinates at $p_{0}$ and $f\left(p_{0}\right)$, respectively, we may assume that $p_{0}=0$ and $f\left(p_{0}\right)=0$ and that $r=\operatorname{Im} z_{n}+h\left(\operatorname{Re} z_{n}, z^{\prime}\right)$ and $\rho=\operatorname{Im} w_{n}+\left(\operatorname{Re} w_{n}, w^{\prime}\right)$ where $h(0)=0, \nabla h(0)=0, g(0)=0, \nabla g(0)=0$, and $z^{\prime}, w^{\prime} \in \mathbf{C}^{\mathbf{n}-1}$. We see from Lemma 12 that at $p_{0}=0$

$$
L_{\rho} \circ f\left(f^{\prime}(0) v, \overline{f^{\prime}(0) v}\right)=\frac{\partial f_{n}}{\partial z_{n}}(0) L_{r}(v, \bar{v})
$$

where $v \in T_{0}^{C} M_{1}, f=\left(f_{1}, \ldots, f_{n}\right)$, and $L_{\rho} \circ f$ is the Levi form of $M_{2}$ at $f(0)=0$. If $M_{2}$ is Levi flat, then we conclude from the above identity that $\partial f_{n}(0) / \partial z_{n}=0$ and by moving $p_{0}$ slightly we may have $\partial f_{n}(z) / \partial z_{n} \equiv 0$ for $z \in U$. Therefore, $f_{n}(z)$ is independent of $z_{n}$. To show $f_{n}(z) \equiv 0$, we consider on $U$

$$
\operatorname{Im} f_{n}\left(z^{\prime}\right)+g\left(\operatorname{Re} f_{n}\left(z^{\prime}\right), f_{1}(z), \ldots, f_{n-1}(z)\right)=0
$$

and take the complex tangential derivative $L_{k}$ as defined in the proof of Lemma 12 to conclude that $f_{n}(z) \equiv 0$. This completes the proof of Theorem 9 .

Proof of Theorem 10. If $M_{2}$ is Levi flat, we choose a point $p \in M_{1}$ such that $M_{1}$ does not contain any complex hypersurface through $p$ and then, by a theorem of Trepeau [T], we have that $f$ extend holomorphically to one side of $M_{1}$.

By Theorem 9 we have $f\left(M_{1} \cup M_{1}^{-}\right) \subset M_{2}$ where $M_{1}^{-}$is one side of $M_{1}$. But this contradicts the following fact, which is related to a fact in [P].

Lemma 13. Let $f: M_{1} \rightarrow M_{2}$ be a continuous $\mathrm{CR}$ homeomorphism between $C^{2}$ smooth real hypersurfaces that extends holomorphically to one side of $M_{1}$, say $M_{1}^{-}$; then we have $f\left(M_{1} \cup M_{1}^{-}\right) \not \subset M_{2}$.

Proof. Take a sequence $z_{k} \rightarrow p \in M_{1}, z_{k} \in M-1^{-}$; and consider the sets

$$
E_{k}=\left\{z \in M_{1}^{-}: f(z)=f\left(z_{k}\right)\right\} \text {. }
$$


If $f\left(M_{1} \cup M_{1}^{-}\right) \subset M_{2}$, then Rank $f<n$ everywhere and $E_{k}$ are analytic sets in $M_{1}^{-}$of dimension $\geq 1$. Each $E_{k}$ has no more than one limit on $M_{1}$ because $f$ is homeomorphic in $M_{1}$. By Shiffman's theorem [S], $\bar{E}_{k}$ are analytic sets in $M_{1}^{-}$. We have $d\left(p, \bar{E}_{k}\right) \rightarrow 0$; and since $f: M_{1} \rightarrow M_{2}$ is a homeomorphism,

$$
\lim d\left(p^{\prime}, \bar{E}_{k}\right)>0
$$

for any other point $p^{\prime} \neq p$ in $M_{1}$ (here we denote by $d\left(p, \bar{E}_{k}\right)$ the distance between $p$ and $\bar{E}_{k}$ ). By the continuity principle, $f$ extends holomorphically through the point $p$. We obtain a contradiction because the restriction of $f$ to $M_{1}$ cannot be one-to-one since the rank of $f$ is $n-1$ in a neighborhood of $p$ in $\mathbf{C}^{\mathbf{n}}$.

\section{Proof of THEOREM 1}

In this section we give a proof of Theorem 1. From now on we assume that $f: M_{1} \rightarrow M_{2}$ is a smooth homeomorphic CR mapping between real analytic hypersurfaces in $\mathrm{C}^{2}$. If $M_{1}$ is not Levi flat, i.e., the Levi form of $M_{1}$ does not vanish identically on $M_{1}$, it is well known that if $p \in M_{1}$, then either $M_{1}$ is of finite type at $p$ or of infinite type. In the case of infinite type, $M_{1}$ contains a complex curve through $p$. By our remark in the introduction, it suffices to prove that the transversal component of $f$ does not vanish to infinite order at any point of $M_{1}$. We assume that $f=\left(f_{1}, f_{2}\right)$ where $f_{2}$ is the transversal component of $f$ and $z=\left(z_{1}, z_{2}\right)$ where $z_{2}$ is the complex normal direction of $M_{1}$ at $p$.

Case 1. $M_{1}$ is of finite type at $p$. By a theorem of Pincuk [P], since $M_{1}$ is minimal at $p$ and $f: M_{1} \rightarrow M_{2}$ is a homeomorphic CR mapping, then the inverse $f^{-1}$ of $f$ is also $\mathrm{CR}$ and $f(p)$ is a minimal point of $M_{2}$. If $M_{2}$ is minimally convex at $f(p)$ as defined in [BR1], then the generalized Hopf lemma of Baouendi and Rothschild shows $\frac{\partial f_{2}}{\partial z_{2}}(p) \neq 0$, and therefore the general reflection principle implies that $f$ extends holomorphically to a neighborhood of $p$ in $\mathbf{C}^{2}$.

If $M_{2}$ is not minimally convex at $f(p)$, by a theorem in [BR 1 ], $f^{-1}$, as a CR mapping, extends holomorphically to a neighborhood of $f(p)$ in $\mathbf{C}^{2}$. Hence, the map $f^{-1} \circ f$ is well defined in $M_{1}^{-}$, one side of $M_{1}$, and is identity on $M_{1}$. By the uniqueness of holomorphic functions, we have $f^{-1} \circ f=$ identity in $M_{1}^{-} \cup M_{1}$. Then $f$ is a diffeomorphism. So Theorem 2 applies and we are done in this case too.

Case 2. $M_{1}$ is of infinite type at $p$. If $M_{1}$ is of infinite type at $p$, then by Theorem 10, $M_{2}$ cannot be Levi flat. On the other hand, since $M_{1}$ is real analytic, there is a complex curve passing through $p$, and therefore the image of the curve under $f$ is also a complex curve in $M_{2}$ passing through $f(p)$ since $f$ is a CR homeomorphism. This shows that $M_{2}$ is of infinite type at $f(p)$. If $f_{2}$ vanishes to infinite order at $p$, then by Theorem 4 we must have

$$
f\left(M_{1}^{-} \cup M_{1}\right) \subset M_{2} \text {. }
$$

But this is impossible by Lemma 13. So we have proved that $f_{2}$ vanishes to finite order, and therefore Theorem 2 applies to conclude that $f$ extends 
holomorphically to a neighborhood of $p$ in $\mathbf{C}^{2}$. Combined, this completes the proof of Theorem 1.

\section{ACKNOWLEDGMENT}

The author thanks Professor S. Bell. D. Barrett, and D. A. Taylor for their support and encouragement.

\section{REFERENCES}

[B] S. Bell, Local regularity of CR homeomorphisms, Duke. Math. J. 57 (1988), 295-300.

[BL] S. Bell and L. Lempert, $A C^{\infty}$ Schwartz reflection principle in one and several complex variables, J. Differential Geom. 23 (1990), 899-915.

[BC] S. Bell and D. Catlin, Regularity of CR mappings, Math. Z. 199 (1988), 357-368.

[BR1] S. Baouendi and L. Rothschild, $A$ generalized complex Hopf lemma and its applications to CR mappings, Invent. Math. 111 (1993), 231-348.

[BR2] - A general reflection principle in $\mathrm{C}^{2}$, J. Funct. Anal. 99 (1992), 409-442.

[HKMP] X. Huang, S. Krantz, D. Ma, and Y. Pan, A Hopf lemma of holomorphic functions and applications, Complex Variables Theory Appl. (to appear).

[P] S. Pincuk, CR transformations of real manifolds in $\mathbf{C}^{n}$, Indiana Univ. Math. J. 41 (1992), 1-16.

[S] B. Shiffman, On the removal of singularity analytic sets, Michigan Math. J. 15 (1968), 111-120.

[T] J. Trepreau, Sur le prolongment holomorphe des fonctions $C R$ definies sur une hypersurface reele de classe $\mathrm{C}^{2}$ dans $\mathrm{C}^{n}$, Invent. Math. 83 (1986), 583-592.

Department of Mathematics, Indiana-PuRdue University at Fort Wayne, Fort Wayne, INDIANA 46805 